\title{
Motivação dos homens na busca por assistência prestada pelas estratégias de saúde da família
}

RESUMO | Objetivo: investigar a motivação dos homens na busca por assistência prestada pela Estratégia Saúde da Família. Método: estudo descritivo, exploratório, com abordagem quantitativa, realizada em uma equipe de saúde da família, de modo que a amostra compreendeu 30 homens cadastrados na área de abrangência. Foi utilizado um questionário semiestruturado cujos dados foram analisados conforme epidemiologia descritiva. Resultados: trata-se de homens entre 20 e 74 anos; casados; Ensino Fundamental; predomínio da profissão de pedreiro os quais $67 \%$ trabalham no período diurno. Dos serviços procurados, $68 \%$ correspondem à Medicina cuja motivação da procura foi por consultas e exames. Quanto aos fatores dificultadores, destacase a resistência dos homens; a falta de tempo decorrente da dedicação ao trabalho; e ao comodismo. Conclusão: a motivação dos homens pela procura ao serviço ocorreu por ações de cunho curativo com predomínio de queixas agudas na qual as práticas preventivas não fazem parte do cotidiano.

Palavras-chaves: Masculinidade; Saúde do homem; Atenção primária à saúde.

\begin{abstract}
Objective: to investigate the motivation of men in search of assistance provided by the Family Health Strategy. Method: a descriptive study, exploratory, with a quantitative approach, performed in a family health team in which the sample included 30 men registered in the coverage area. A semi-structured questionnaire was used in which the data were analyzed according to descriptive epidemiology. Results: these are men between 20-74 years; married; elementary school; predominance mason profession in which $67 \%$ work in the daytime. Of popular services, $68 \%$ correspond to medicine whose motivation demand was for consultations and exam. As for complicating factors, there is the strength of men; lack of time due to the hard work; and convenience. Conclusion: the motivation of men by demand service occurred curative nature of actions with a predominance of acute complaints where preventive practices are not part of everyday life.
\end{abstract}

Keywords: Masculinity; Men's health; Primary health care.

RESUMEN | Objetivo: investigar la motivación masculina en busca de asistencia en la Estrategia Salud de la Familia. Método: estudio descriptivo, exploratorio, con abordaje cuantitativo, realizado en un equipo de salud de la familia, la muestra incluía 30 hombres registrados en el área. Se utilizó un cuestionario semiestructurado en el que se analizaron los datos por medio de epidemiología descriptiva. Resultados: hombres entre 20-74 años; casado; enseñanza fundamental; predominio de albañilesque trabajan durante el día. De los servicios buscados, $68 \%$ corresponde a la medicina cuya motivación fue las consultas y exámenes. Cuanto a los factores de complicación, se destaca la resistenciamasculina; la falta de tiempo debido a dedicación del trabajo; y la comodidad. Conclusión: la motivación masculina por la demanda del servicio se produjo la naturaleza curativa de las acciones con un predominio de las quejas agudas en las que las prácticas preventivas no son parte de la vida cotidiana.

Palabras claves: Masculinidad; Salud del hombre; Atención primaria de salud.

\section{Patrick Leonardo Nogueira da Silva}

Enfermeiro, Mestrando pelo Programa de Pós-Graduação em Cuidado Primário em Saúde da Universidade Estadual de Montes Claros (PPGCPS/UNIMONTES). Montes Claros, MG, Brasil.

ORCID: 0000-0003-2399-9526

\section{Emily Loyara Grilo Silva}

Enfermeira pelas Faculdades Unidas do Norte de Minas (FUNORTE). Montes Claros, MG, Brasil.

ORCID: 0000-0002-7699-3331

\section{Veridiana Martins Santos}

Enfermeira pelas Faculdades Unidas do Norte de Minas (FUNORTE). Montes Claros, MG, Brasil.

ORCID: 0000-0001-5209-7922

Recebido em: 28/08/2020

Aprovado em: 09/11/2020

\section{Ana Patrícia Fonseca Coelho Galvão}

Enfermeira, Mestre em Saúde e Ambiente, Professora do Departamento de Enfermagem da Universidade Ceuma (UNICEUMA). São Luís, MA, Brasil.

ORCID: 0000-0003-3376-5678

\section{Valdira Vieira de Oliveira}

Enfermeira, Mestre em Enfermagem, Professora do Departamento de Enfermagem da Faculdade Santo Agostinho (FASA). Montes Claros, MG, Brasil.

ORCID: 0000-0003-2020-2489

\section{Carolina dos Reis Alves}

Enfermeira, Doutora em Ciências da Saúde, Professora do Departamento de Enfermagem da Faculdade Santo Agostinho (FASA). Montes Claros, MG, Brasil.

ORCID: 0000-0003-2107-6306
INTRODUÇÃO

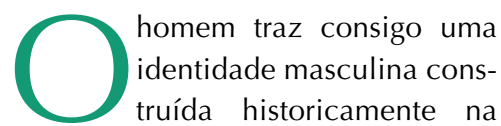
qual envolve papéis sociais, diferenciando-o do gênero feminino. Ser homem está relacionado à invulnerabilidade, força e virilidade e isso se relaciona à desvalorização ao seu autocuidado em que os homens revelam maior dificuldade de buscar por assistência em saúde em razão de sua autopercepção que a procura de cuidados é uma tarefa do gênero feminino. Dessa forma, demandar cuidados de saúde é algo que desmerece os sujeitos criados para assistir e promover legítimos representantes do gênero masculino. Essa imagem masculina do "ser forte" pode acarretar em práticas 
de pouco cuidado com o próprio corpo, de modo a tornar o homem vulnerável a uma série de situações ${ }^{(1,2,3)}$.

Os indicadores de morbimortalidade do nosso país têm traçado um perfil que se mantém há anos, no qual as mulheres são mais acometidas por problemas de saúde, buscam mais consultas médicas, consomem mais medicamentos e se submetem a mais exames que os homens. No entanto há um maior número de homens internados em situações graves, como também a procura por serviços de emergência e a taxa de mortalidade por causas patológicas graves é maior entre os homens ${ }^{(4)}$. Os serviços de saúde têm uma deficiência em absorver a demanda apresentada pelos homens, proporcionada pela organização dos serviços que não estimula o acesso destes homens e, pelo fato das próprias campanhas de saúde pública não se voltarem para este segmento da população. Assim, são necessárias mudanças nas estratégicas dos serviços de saúde e no enfoque relacionado ao gênero masculino ${ }^{(1)}$.

Por um longo período, a saúde do homem se mostrou carente de uma política de incentivo que vise à prevenção de doenças e a promoção de saúde. Levando em consideração o alto índice de doenças crônicas detectadas em homens somada com a elevada estatística de morte em adultos jovens, foi lançado pelo Ministério da Saúde (MS), em agosto de 2009, a Política Nacional de Atenção Integral à Saúde do Homem (PNAISH) que visa incentivar os homens a procurar uma mudança cultural, já que, em geral, os homens só recorrem aos serviços quando já estãodoentes, tendo que ser atendidos por especialistas, o que gera um aumento de gastos públicos ${ }^{(5)}$.

A PNAISH visa qualificar a atenção à saúde da população masculina na perspectiva de linhas de cuidado. O reconhecimento de que a população masculina acessa o sistema de saúde por meio da atenção especializada e requer mecanismos de fortalecimento e qualificação da Atenção Primária à Saúde (APS), para que a atenção à saúde não se restrinja à recuperação, garantindo, sobretudo, a promoção da saúde e a prevenção a agravos ${ }^{(5,6)}$. A mobilização da população masculina brasileira para a luta pela garantia de seu direito social à saúde é um dos desafios dessa política, que pretende sensibilizar homens para o reconhecimento e a enunciação de suas condições sociais e de saúde, para que advenham sujeitos protagonistas de suas demandas, consolidando seu exercício e gozo dos direitos de cidadania ${ }^{(6)}$.

A realização deste estudo pode ser justificada tendo em vista a baixa procura e adesão do homem em caráter preventivo aos serviços de saúde, principalmente na APS. Muitas das vezes, a procura do homem por serviços assistenciais é de caráter urgente decorrente do surgimento sintomático de uma determinada patologia já instalada ou até mesmo pela sua progressão culminando na piora do seu prognóstico. Sendo assim, o estudo abordou a seguinte questão norteadora: qual a motivação dos homens na busca pela assistência prestada pela Estratégia Saúde da Família (ESF)?

Portanto, objetivou-se investigar a motivação dos homens na busca por assistência prestada pela ESF.

\section{MÉTODOS}

Artigo da monografia intitulada "A motivação dos homens na busca por assistência prestada na Estratégia Saúde da Família", apresentada ao Departamento de Enfermagem das Faculdades Unidas do Norte de Minas/FUNORTE. Montes Claros, Minas Gerais, Brasil. 2013.

Trata-se de um estudo descritivo, exploratório, com abordagem quantitativa, realizada nas ESF dos bairros Village do Lago, Clarice Athaide e Jaraguá 1 na qual apresenta 2593 habitantes cadastrados na Unidade Básica de Saúde (UBS), sendo localizada na região norte do município de Montes Claros, Minas Gerais. A amostra do estudo foi constituída por 30 homens desta área de abrangência.
Foi enviada uma carta de apresentação, juntamente com uma cópia do projeto de pesquisa, e um Termo de Consentimento Institucional (TCI) à Coordenação da APS da Secretaria Municipal de Saúde da cidade de Montes Claros (SMS-MOC) para autorização do estudo. A instituição foi devidamente orientada quanto às diretrizes da pesquisa e autorizou a realização do estudo por meio da assinatura do $\mathrm{TCl}$ de modo a estar ciente. A coleta de dados foi realizada no segundo semestre de 2013, durante o mês de outubro, pelo pesquisador responsável.

Foram adotados os seguintes critérios de inclusão para participação no estudo: (1) estar cadastrado na Ficha A; (2) ser encontrado na UBS durante o horário de atendimento em dias e horários diferentes; (3) ter mais de 20 anos; (4) ter condições cognitivas de responder a entrevista; e (5) aceitar participar da pesquisa.

Foi utilizado um questionário semiestruturado, de elaboração própria e validado por meio de um pré-teste, como instrumento de coleta de dados. O pré-teste foi realizado no mês de setembro de 2013 com outros homens da mesma área de abrangência cuja escolha foi aleatória e cujo qual não compôs a amostra do estudo. Sua realização objetivou avaliar a viabilidade de aplicação das variáveis do questionário de pesquisa. Após a coleta de dados, os mesmos foram armazenados e tabulados por meio do programa Statistical Package for the Social Sciences (SPSS), versão SPSS $®$ for Windows 15, e representados em uma tabela de modo a apresentar freqüências absolutas (n), percentuais (\%) e medidas de tendência central (MTC), sendo elas: média aritmética (MA), moda (Mo), mediana (Me), média aritmética ponderada (MAP) e desvio padrão (DP). A análise dos dados se deu por meio de epidemiologia descritiva simples não-paramétrica e não-probabilística.

O estudo obedeceu aos preceitos éticos estabelecidos pela Resolução $n^{\circ} 466$, de 12 de dezembro de 2012, do ConseIho Nacional de Saúde (CNS), na qual regulamenta a realização de pesquisa en- 
volvendo seres humanos ${ }^{(7)}$. O projeto de pesquisa foi apreciado e aprovado pelo Comitê de Ética em Pesquisa das Faculdades Unidas do Norte de Minas (CEP FUNORTE), com o parecer consubstanciado $\mathrm{n}^{\circ}$ 235.948/2013, Certificado de Apresentação para Apreciação Ética (CAAE) $n^{\circ}$ 13330813.9.0000.5141. Os participantes foram orientados quanto às diretrizes do estudo e assinaram o Termo de Consentimento Livre e Esclarecido (TCLE).

\section{RESULTADOS}

A amostra foi composta por homens de 20 a 74 anos, com prevalência da faixa etária de 40 a 60 anos (46,6\%). No que se refere ao estado civil, houve um predomínio de homens casados (50\%). Quanto à escolaridade, a maioria apresenta o Ensino Médio (41\%). Destes, 18\% não concluiu o Ensino Médio e 23\% concluíram. A maior parte dos homens era pedreiro $(20,1 \%)$ e trabalhavam no período diurno $(66,7 \%)$, fator que dificulta o acesso do homem ao serviço de saúde, tendo em vista que o horário de funcionamento do mesmo é no período matutino e vespertino. O serviço mais procurado na UBS é o atendimento médico $(66,7 \%)$ na qual a motivação da procura se deve, em sua maior parte, a consultas (53,3\%) e exames $(40 \%)$. Em se tratando dos fatores dificultadores ao acesso do homem na UBS, prevaleceu a resistência masculina e o trabalho $(40 \%$ e $26,6 \%$, respectivamente). Verificou-se, ainda, que a periodicidade da procura dos homens, em sua maioria, compreendeu um período menor que seis meses (63,3\%). Observou-se que a maior parte dos homens $(93,3 \%)$ procura o serviço de saúde pelo menos uma vez ao ano (Tabela 1).

\section{DISCUSSÃO}

Neste estudo, prevaleceu adultos jovens com faixa etária entre 40-60 anos. A idade configura um fator de risco não modificável à aquisição de várias doenças crônicas. O adulto jovem, tendo este
Tabela 1 - Perfil socioeconômico e do acesso ao serviço de saúde de homens cadastrados na ESF. Montes Claros (MG), 2013.

\begin{tabular}{lcccccc}
\multicolumn{1}{c}{$\begin{array}{c}\text { Variável } \\
\text { Faixa etária (anos) }\end{array}$} & $\mathrm{n}=30$ & $\%$ & $\mathrm{MA}$ & Mo & Me & MAP \pm DP \\
\hline $20-39$ & 12 & 40,0 & & 30 & 30 & $30,08 \pm 5,599$ \\
$40-59$ & 14 & 46,6 & 42,86 & 44 & 46,5 & $47,50 \pm 5,774$ \\
$\geq 60$ & 04 & 13,4 & & 65 & 65 & $65,00 \pm 4,082$
\end{tabular}

\section{Estado civil}

\begin{tabular}{lllllll} 
Solteiros & 12 & 40,0 & - & - & - \\
Casados/União estável & 15 & 50,0 & - & - & - & - \\
\hline Divorciados/Desquitados & 03 & 10,0 & & - & - & -
\end{tabular}

Escolaridade

\begin{tabular}{lcccccc} 
Analfabeto & 04 & 13,3 & - & - & - \\
Ensino Fundamental & 08 & 26,6 & - & - & - \\
\hline Ensino Médio & 12 & 40 & - & - & - & - \\
Ensino Superior & 00 & 00 & & - & - & - \\
Não respondeu & 06 & 20,1 & - & - & -
\end{tabular}

Profissão

\begin{tabular}{|c|c|c|c|c|c|c|}
\hline Pedreiro & 06 & 20,1 & \multirow{10}{*}{-} & - & - & - \\
\hline Servente de pedreiro & 04 & 13,4 & & - & - & - \\
\hline Vendedor & 02 & 6,7 & & - & - & - \\
\hline Comerciante & 02 & 6,7 & & - & - & - \\
\hline Eletricista & 01 & 3,1 & & - & - & - \\
\hline Operador de caldeira & 01 & 3,1 & & - & - & - \\
\hline Lavrador & 04 & 13,4 & & - & - & - \\
\hline Vigia & 02 & 6,7 & & - & - & - \\
\hline Auxiliar de coleta & 02 & 6,7 & & - & - & - \\
\hline Serviços gerais & 02 & 6,7 & & - & - & - \\
\hline Estudante & 04 & 13,4 & & - & - & - \\
\hline
\end{tabular}

\section{Horário de trabalho}

\begin{tabular}{|c|c|c|c|c|c|c|}
\hline $6 \mathrm{~h}$ às $18 \mathrm{hs}$ & 20 & 66,7 & \multirow[b]{2}{*}{12,45} & 14 & 13 & $12,50 \pm 3,720$ \\
\hline $19 \mathrm{hs}$ às $5 \mathrm{~h}$ & 02 & 6,7 & & - & 12 & $12,00 \pm 9,899$ \\
\hline Outros & 08 & 26.6 & & 11 & 11 & $11,75 \pm 3,195$ \\
\hline
\end{tabular}

\section{Serviço procurado}

\begin{tabular}{|c|c|c|c|c|c|c|}
\hline Medicina & 20 & 66,7 & \multirow{3}{*}{-} & - & - & - \\
\hline Enfermagem & 09 & 30,0 & & - & - & - \\
\hline Odontologia & 01 & 3,3 & & - & - & - \\
\hline \multicolumn{7}{|c|}{ Motivação da procura } \\
\hline Consultas & 16 & 53,3 & \multirow{3}{*}{-} & - & - & - \\
\hline Exames & 12 & 40,0 & & - & - & - \\
\hline Imunização & 02 & 6,7 & & - & - & - \\
\hline
\end{tabular}

Fatores dificultadores 
Resistência

\begin{tabular}{lll} 
Trabalho & 08 & 26,6 \\
\hline Comodismo & 06 & 20,0 \\
\hline Sem dificuldade & 04 & 13,4
\end{tabular}

Frequência da procura (meses)

\begin{tabular}{|c|c|c|c|c|c|c|}
$<6$ & 19 & 63,3 & & 4 & 4 & $3,47 \pm 1,348$ \\
\hline $6-12$ & 09 & 30,0 & 6,16 & $6 / 8$ & 8 & $8,55 \pm 2,127$ \\
\hline$>12$ & 02 & 6,7 & & - & 21 & $21,00 \pm 4,242$ \\
\hline
\end{tabular}

Fonte: Os autores. Montes Claros (MG), Brasil.

$\mathrm{MA}=$ Média Aritmética. Mo = Moda. $\mathrm{Me}=$ Mediana. $\mathrm{MAP}=$ Média Aritmética Ponderada. $\mathrm{DP}=$ Desvio Padrão.

idade entre 20-59 anos, principalmente no sexo masculino, está mais propenso ao desenvolvimento destas doenças com o passar do tempo e conforme o aumento de outros fatores de risco ${ }^{(8,9)}$. O estado civil é outro fator importante no cuidado da saúde masculina, pois homens casados, diferentemente de homens solteiros ou divorciados, tendem a se cuidar por influência de seu cônjuge. O dado encontrado nessa pesquisa é similar ao observado no estudo realizado em Campina Grande, Paraíba (PB), em que constatou que a maioria dos sujeitos é de homens casados ou convivem com outra pessoa ${ }^{(9)}$.

Dentro da amostra estudada, o estado civilnão se correlaciona com a procura/ não procura pelos serviços de saúde, pois os homens casados não se sobrepõem significativamente aos solteiros. Dessa forma, não confirmam os resultados de outras pesquisas, realizadas em outros contextos culturais que afirmam que os homens casados ou com convivência com uma parceira procuram mais o médico do que os homens solteiros ${ }^{(10)}$.

A escolaridade tem influência na busca do serviço de saúde. Observou-se que a maior parte da amostra deste estudo relatou o ensino médio como maior nível de escolaridade. Destes, prevaleceu o ensino médio completo. Dos participantes do estudo, nenhum cursou o ensino superior. $\mathrm{O}$ aperfeiçoamento das informações estimula a busca no cuidado à saúde de modo a gerar ações pre- ventivas para si e para outras pessoas. Ter o conhecimento atualizado não é sinônimo de estar com a saúde em condições satisfatórias, pois muitos homens não colocam em prática aquilo que aprendem tendo em vista a resistência masculina na melhoria de sua qualidade de vida (QV). Os dados deste estudo corroboramcom outro estudioso na qual afirma que, mesmo os homens que apresentam curso superior e tem o conhecimento corretosobre a temática abordada, os mesmos não o colocam em prática ${ }^{(1)}$.

A profissão de pedreiro foi a mais declarada pelos homens entrevistados, de modo a revelar que os sujeitos do estudo fazem parte de categorias profissionais autônomas na qual na há vínculo empregatício. Sendo assim, a ausência de um emprego estávelpode gera diminuição do rendimento e sustento familiar e dificultar a busca ao serviço de saúde para ações de cunho promocional e preventivo. Além disso, a sociedade contribui para a questão do gênero masculino, pois, historicamente, só o homem podia trabaIhar de modo a ser o provedor da casa e o principal responsável pelo sustento familiar, enquanto que as mulheres eram responsáveis pelo serviço doméstico ${ }^{(11)}$.

Em se tratando do horário de trabaIho, este caracteriza um obstáculo para a busca pelo serviço de saúde dentro do público masculino, pois o horário de funcionamento da UBS, sendo esse das $7 \mathrm{~h}$ da manhã às $17 \mathrm{hs}$ da tarde, coincide com o horário de trabalho da maior parte dos homens, de modo que a UBS não se encontrará em funcionamento durante a noite. Neste estudo, uma mínima fração da amostra desempenha suas atividades laborais no período noturno. Observa-se, ainda, que outra parte da amostra não trabalha em período integral ou noturno, mas sim durante meio período, podendo ser apenas durante o turno matutino ou vespertino, e ainda sim não buscarem pelo serviço de saúde. Em outro estudo é corroborado que um dos motivos da não procura dos homens aos serviços de saúde é que o agendamento de consultas e exames é no mesmo horário de trabalho dos mesmos ${ }^{(12)}$.

Dos serviços disponibilizados ao público pela UBS, houve um predomínio da procura por atendimento médico. Ressalta-se que a consulta médica está voltada para ações curativas, enquanto que a enfermagem volta-se à promoção da saúde e prevenção de agravos, sendo mais demorada a consulta de enfermagem, pois aborda educação para mudança de hábitos que possam colocar em risco a saúde do indivíduo. Com isso, os homens trazem consigo uma característica em comum, sendo esta a pressa para irem embora por medo de perderem o trabaIho, medo de terem um diagnóstico ou prognóstico ruim, a questão da masculinidade, machismo ao associar a buscapor serviços de saúde com fragilidade, já que o homem deve ser sempre modelo de força, virilidade e invulnerabilidade. Por isso, o homem não pode adoecer ou passar o tempo todo nas UBS participando de ações promocionais e preventivas ${ }^{(13)}$.

A motivação do homem na busca pelo serviço de saúde muitas vezes é de cunho curativo, visando à realização de consultas médicas e exames em sua maior parte, tendo em vista a manifestação sintomática de uma patologia já instalada. Salienta-se que uma pequena parcela da amostra se preocupa em prevenir de modo a evitar o tratamento farmacológico. Esse dado ratifica que os homens deste estudo procuram o serviço 
de saúde apenas quando estão realmente doentes. Outro estudo constatou que, por questão cultural, o homem é mais objetivo ao procurar o serviço de modo a apresentar motivações curativas - tratamento de doença, atestado, exame para trabalho - sempre com a justificativa de que não têm tempo a perder ${ }^{(2)}$. Um aspecto relevante do estudo é que $6,7 \%$ dos homens que procuraram por ações preventivas são homens idosos que, em virtude da imunizaçãocontra a Influenza, encontra-se mais presente no serviço de saúde.

A APS, porta de entrada principal aos serviços de saúde, é marcadamente dirigida aos problemas de saúde das mulheres, pois prevenção e cuidado em saúde são socialmente caracterizados como tarefas essencialmente femininas $^{(14)}$. Os motivos que levam os homens ao serviço de saúde são: acidente, dor, virose, conjuntivite, hipertensão e infecção de pele, ou seja, a maioria vai à UBS apenas quando precisa de ações curativas e em nenhum dos casos participam de grupos para promoção da saúde e prevenção de doenças. Este dado corrobora com o estudo de outro pesquisador na qual afirma que a grande maioria dos homens só procura o serviço de saúde apenas em caso de enfermidades graves, acidentes ou impossibilidade física ${ }^{(1)}$, reafirmando o disposto pelos documentos do MS e outros estudos na qual os homens só procuram o serviço de saúde quando já estão doentes ${ }^{(5,6,9)}$. Isso ocorre porque existe uma cultura difundida em nossa sociedade de que o homem é um ser dominador, invencível e que, portanto, não sente dor, assim a masculinidade acaba sendo o principal fator do aumento da mortalidade entre os homens.

Dos fatores dificultadores abordados pelos homens neste estudo, a própria resistência prevaleceu nos resultados, seguida da dedicação pelo trabalho. Sendo assim, estes homens vão deixando o tempo passar e se automedicando de modo a não se preocupar em cuidar da saúde e procurar atendimento preventivo. Quando vão ao serviço de saúde pressupõem-se o agravamento do quadro clínico. Esta prerrogativa encontra-se em conformidade com outros estudos ${ }^{(15)}$. Observou-se que $13,4 \%$ disseram que para eles não há nenhuma dificuldade em procurar o serviço de saúde. Enfatizam que a jornada de trabalho, a falta de tempo, a impossibilidade de deixar as atividades não são empecilhos na busca pelos serviços de $\operatorname{APS}^{(1,10,13,16)}$. No Brasil, é bastante disseminada a ideia de que as unidades de APS são serviços destinados quase que exclusivamente paracrianças, mulheres e idosos. Muitas são as justificativas para pouca procura ou até mesmo ausência dos homens nos serviços de saúde. Essa resistência do homem em se cuidar não está associada apenas às condições sociais ou a época, e sim a uma cultura em que os homens são condicionados como seres fortes e resistentes quando comparados às mulheres ${ }^{(17)}$.

Um dos fatores que pode contribuir para a resistência ou comodismo é a organização do serviço não estar direcionada para as necessidades do público masculino, ambiente mais atrativo para o publico feminino e infantil gerando, portanto, a pouca procura masculina. $\mathrm{O}$ homem, de um modo geral, demonstra se preocupar mais com o trabalho do que com a própria saúde e prefere trabalhar doente a gerar absenteísmo, pois a ausência do trabalho não é bem avaliada pelo empregador e o mesmo precisa garantir o sustento da casa e dos filhos, pois o gênero masculino é o responsável por ser o provedor da família. O medo de ser demitido após a comunicação que está doente e especialmente uma enfermidade crônica, ocasionando a busca do serviço apenas por questões agudas como a dor e ações curativas ${ }^{(9)}$.

Mais da metade dos homens procuraram o serviço de saúde com periodicidade menor que seis meses. Neste estudo, é possível inferir conforme os resultados que a maior parte dos homens procura a UBS pelo menos uma vez no ano. Este fato é corroborado em outro estudo na qual afirma que, para que haja uma 
adesão dos homens com idade entre 20 e 59 anos, além de se beneficiarem da PNAISH, é preciso ainda que os mesmos busquem os serviços pelo menos uma vez ao ano, mas não necessariamente nos períodos de campanha ${ }^{(18)}$.

\section{CONCLUSÃO}

Este estudo demonstrou que a ESF, localizada na região oeste, atendeu a recomendação da PNAISH, entretanto não atendeu às diretrizes da APS, em especial, a Saúde da Família que tem como objetivo principal as ações de promoção e prevenção, pois a motivação dos homens pela procura ao serviço de saúde ocorreu por ações de cunho curativo com predomínio de queixas agudas. Diversos aspectos relacionados ao universo masculino e seus cuidados com a saúde foram analisados para fins de explicar a pouca procura dos homens pelos serviços de saúde, dentre eles destacam-se a resistência dos homens associada à priorização ao trabalho, sendo necessário que exista uma flexibilidade do horário para atender ao público trabalhador e garantindo a acessibilidade funcional.

Como limitação do estudo, destaca-se a quantidade amostral reduzida decorrente da incompatibilidade de horário dos homens na ESF para o estudo de modo a dificultar a realização de uma análise mais aprofundada. Nas ESF se fazem necessárias reflexões e problematizações que contribuam para a criação de mecanismos para a melhoria da atenção dispensada aos homens. Foram verificadas que as práticas preventivas, por variados motivos, sejam elas de ordem estrutural ou cultural, não fazem parte do cotidiano dessa população, fato este justificado pela concepção de que ser homem significa invulnerabilidade, força e virilidade e que a procura pelo serviço de saúde fere a sua masculinidade. Portanto, em termos de lacunas, pode-sedestacar que pesquisas ainda se fazem necessáriaspara que sejam aprofundadas as relações entre masculinidade e saúde.

\section{Referências}

1. Bibiano AMB, Moreira RS, Tenório MMGO, Silva VL. Fatores associados à utilização dos serviços de saúde por homens idosos: uma revisão sistemática da literatura. Ciênc. Saúde Colet. [Internet]. 2019 [cited 2020 Jun 20];24(6):226378. doi: https://doi.org/10.1590/1413-81232018246.19552017

2. Lemos AP, Ribeiro C, Fernandes J, Bernardes K, Fernandes R. Saúde do homem: os motivos da procura dos homens pelos serviços de saúde. Rev. Enferm. UFPE on line [Internet]. 2017 [cited 2020 Jun 20];11(Supl. 11):4546-53. doi: http://dx.doi.org/10.5205/reuol.11138-99362-1-SM.1111sup201714

3. Trilico MLC, Oliveira GR, Kijimura MY, Pirolo SM. Discursos masculinos sobre prevenção e promoção da saúde do homem. Trab. Educ. Saúde [Internet]. 2015 [cited 2020 Jun 20];13(2):381-95. doi: https://doi.org/10.1590/ 1981-7746-sip00015

4. Brasil. Ministério da Saúde. Secretaria de Atenção à Saúde. Departamento de Ações Programáticas Estratégicas. Perfil da morbimortalidade masculina no Brasil [Internet]. Brasília: Ministério da Saúde, 2018 [cited 2020 Jun 20]. 52 p. https://www.saude.gov.br/images/pdf/2018/novembro/07/Perfil-da-morbimortalidade-masculina-no-Brasil.pdf

5. Coelho EBS, Schwarz E, Bolsoni CC, Conceição TB. Política Nacional de Atenção Integral à Saúde do homem [Internet]. Florianópolis: Universidade Federal de Santa Catarina, 2018 [cited 2020 Jun 20]. 66 p. https://www.saude.gov.br/ images/pdf/2018/novembro/07/livroPol--ticas-2018.pdf

6. Brasil. Ministério da Saúde. Secretaria de Atenção à Saúde. Departamento de Ações Programáticas Estratégicas. Saúde do homem. Brasilia, 2009 [cited 2014 dec 10]. http://portalsaude.saude.gov.br/index.php/o-ministerio/principal/secretarias/805-sas-raiz/daet-raiz/saude-do-homem/11-saude-do-homem/ 12325-apresentacao-saude-homem

7. Brasil. Ministério da Saúde. Conselho Nacional de Saúde. Resolução n 466 de 12 de dezembro de 2012. Aprovam diretrizes e normas regulamentadoras de pesquisas envolvendo seres humanos. Brasília, 2012 [cited 2013 Feb 20]. http://conselho.saude.gov.br/resolucoes/2012/Res0466.pdf

8. Moura EC, Gomes R, Pereira GMC. Percepções sobre a saúde dos homens numa perspectiva relacional de gênero, Brasil, 2014. Ciênc. Saúde Colet. [Internet]. 2017 [cited 2020 Jun 20];22(1):291-300. doi: https://doi. org/10.1590/1413-81232017221.17482015

9. Arruda GO, Mathias TAF, Marcon SS. Prevalência e fatores associados à utilização de serviços públicos de saúde por homens adultos. Ciênc. Saúde Colet. [Internet]. 2017 [cited 2020 Jun 20];22(1):279-90. doi: http://dx.doi. org/10.1590/1413-81232017221.20532015

10. Silva PLN, Maciel MM, Cafersan CS, Santos S. A política de atenção à saúde do homem no Brasil e os desafios da sua implantação: uma revisão integrativa. Enferm. Global [Internet]. 2013 [cited 2015 Mar 19];12(4):414-43. doi: https://doi.org/10.6018/eglobal.12.4.173471

11. Cardos DV, Amorim DS, Robles AJM, Rangel TLV. A invisibilidade dos homens nas unidades de atenção primária à saúde no Brasil de acordo com estudos realizados nos últimos dez anos. In: Anais do IV Seminário Científico da FACIG, III Jornada de Iniciação Científica. Manhuaçu: FACIG, 2018 [cited 2020 Jun 20]. http://pensaracademico.facig.edu.br/index.php/semiariocientifico/article/download/833/733

12. Oliveira MM, Daher DV, Silva JLL, Andrade SSCA. A saúde do homem em questão: busca por atendimento na atenção básica de saúde. Ciênc. Saúde Colet. [Internet]. 2015 [cited 2020 Mar 20];20(1):273-8. doi: https://doi. org/10.1590/1413-81232014201.21732013

13. Pereira MCA, Barros JPP. Públicos masculinos na estratégia de saúde da família: estudo qualitativo em Parnaíba-PI. Psicol. Soc. [Internet]. 2015 [cited 2020 Jun 20];27(3):587-98. doi: https://doi.org/10.1590/1807-03102015v$27 n 3 p 587$

14. Alves BMS, Araújo CJS, Almeida SLS, Guimarães ALS. Atuação do enfermeiro da atenção básica diante das dificuldades para a implementação da política de saúde do homem. Rev. Enferm. UFPE on line [Internet]. 2017 [cited 2020 Jun 20];11(Supl. 12):5391-401. doi: http://dx.doi.org/10.5205/1981-8963-v11i 12a110143p5391-5401-2017

15. Yoshida VC, Andrade MGG. 0 cuidado à saúde na perspectiva de trabalhadores homens portadores de doenças crônicas. Interface (Botucatu) [Internet]. 2016 [cited 2020 Jun 20];20(58):597-610. doi: http://dx.doi.org/10.1590/180757622015.0611

16. Leite JF, Dimenstein M, Paiva R, Carvalho L, Amorim AKMA, França A. Sentidos da Saúde numa Perspectiva de Gênero: um Estudo com Homens da Cidade de Natal/RN. Psicol. Ciênc. Prof. [Internet]. 2016 [cited 2020 Jun 20];36(2):34153. doi: https://doi.org/10.1590/1982-3703001812013

17. Teixeira DBS, Cruz SPL. Atenção à saúde do homem: análise da sua resistência na procura dos serviços de saúde. Rev. Cubana Enferm. [Internet]. 2016 [cited 2020 Jun 20];32(4):1-13. http://www.revenfermeria.sld.cu/index.php/ enf/article/view/985/209

18. Pimentel WC, Vasconcelos ICBL, Ribeiro RRS, Prestes JYN, Silva IL, Albuquerque $\mathrm{MS}$, et al. Avaliação da política de saúde do homem na atenção primária de um município do agreste pernambucano. Braz. J. Health Rev. [Internet]. 2020 [cited 2020 Jun 20];3(3):3935-47. doi: http://dx.doi.org/10.34119/ bjhrv3n3-003 